\title{
Mejores Prácticas de Manejo en el Area Agrícola de los Everglades: Prevención de el Derramamiento de Fertilizantes 1
}

\author{
O.A. Diaz, S.H. Daroub, R.W. Rice, T.A. Lang, V.M. Nadal, and M. Chen ${ }^{2}$
}

\section{Introducción}

Nutrientes de plantas como el fósforo (P) son aplicados a los cultivos para optimizar la productividad y maximizar los beneficios. Sin embargo, la aplicación excesiva de nutrientes y el uso incorrecto de métodos de aplicación puede resultar en el movimiento de nutrientes a aguas subterráneas y superficiales. Además, durante el proceso de fertilización en los campos agrícolas, existe la posibilidad de derramamientos accidentales de fertilizantes que pueden aún más impactar negativamente la calidad de las aguas subterráneas y de superficie.

La prevención de el derramamiento de fertilizantes que contienen $\mathrm{P}$ es una de las Mejores Prácticas de Manejo aprovadas por el Distrito de Manejo de el Agua de el Sur de la Florida. Estas prácticas están diseñadas para reducir las cargas de P en el drenaje de el Area Agrícola de los Everglades (EAA). La prevención de derramamientos de fertilizantes es una de las Mejores Prácticas de Manejo extensamente usadas por los agricultores de esta zona. Esta práctica de manejo es fácil de implementar y puede tener un impacto inmediato en la reducción de cargas de $\mathrm{P}$ proveniente de las fincas. Este documento de EDIS es parte de una serie de publicaciones que proporciona pautas actualizadas para la implementación de las Mejores Prácticas de Manejo comunmente usadas a nivel de fincas y diseñadas para reducir las cargas de $\mathrm{P}$ provenientes de fincas localizadas el EAA.

\section{Prevención de el Derramamiento de Fertilizantes}

El fósforo es uno de los elementos esenciales para el crecimiento de las plantas. Como los suelos agrícolas son a menudo bajos en la cantidad de $\mathrm{P}$ diponibles para las plantas, aplicaciones suplementarias de $\mathrm{P}$ son frecuentamente necesarias para poder mantener una producción de cultivo rentable. Sin embargo, el $\mathrm{P}$ es también considerado como uno de los factores principales que controla la producción primaria subyacente en ecosistemas acuáticos (por ejemplo,la producción de

1. Este documento es SL231-SP, una circular del Departamento de Suelos y Agua, Servicio de Extensión Cooperativa de la Florida, Instituto de Ciencias Agrícolas y Alimentarias, Universidad de la Florida. Fecha de publicación original: Mayo 2007. Visite el sitio web del EDIS en http://edis.ifas.ufl.edu.

2. O.A. Diaz, T.A. Lang, y M. Chen, Investigadores Asociados, V.M. Nadal, Químico Principal, y S.H. Daroub, Profesor Asociado, Centro de Educación e Investigación de los Everglades, Belle Glade, and R.W. Rice, Especialista de Extensien Caña de Az, Condado de Palm Beach; Servicio de Extensión Cooperativa de la Florida, Instituto de Ciencias Agrícolas y Alimentarias, Universidad de la Florida, Gainesville, FL 32611.

The Institute of Food and Agricultural Sciences (IFAS) is an Equal Opportunity Institution authorized to provide research, educational information and other services only to individuals and institutions that function with non-discrimination with respect to race, creed, color, religion, age, disability, sex, sexual orientation, marital status, national origin, political opinions or affiliations. U.S. Department of Agriculture, Cooperative Extension Service, University of Florida, IFAS, Florida A. \& M. University Cooperative Extension Program, and Boards of County Commissioners Cooperating. Larry Arrington, Dean 
organismos fotosintéticos como algas, plantas, etc.). En casos donde el uso inapropiado de fertilizantes fosfóricos conducen a un aumento de los niveles de $\mathrm{P}$ dentro de ecosistemas acuáticos, un excesivo crecimiento de algas y plantas puede ocurrir, lo que contribuye a procesos de eutrificación (degradación ambiental) en las corrientes de aguas, lagos y pantanos. Muchas de las fuentes de fertilizantes fosfóricos utilizados en agricultura se disuelven rápidamente en agua. Tan pronto el $\mathrm{P}$ se disuelve en aguas superficiales, las opciones para removerlo de una forma efectiva son muy limitadas and prohibitivamente costosas (Anderson and Ceric, 1992; Brown and Caldwell, 1993; BION Technologies, 1994). Algunas de las consecuencias directas de las concentraciones excesivas de $\mathrm{P}$ en aguas superficiales son el crecimiento de algas, bajos niveles de oxígeno disuelto, excesivo crecimiento de malezas acuáticas, incremento de la sedimentacion, y mayor turbidez (Figuras 1a y 1 b).

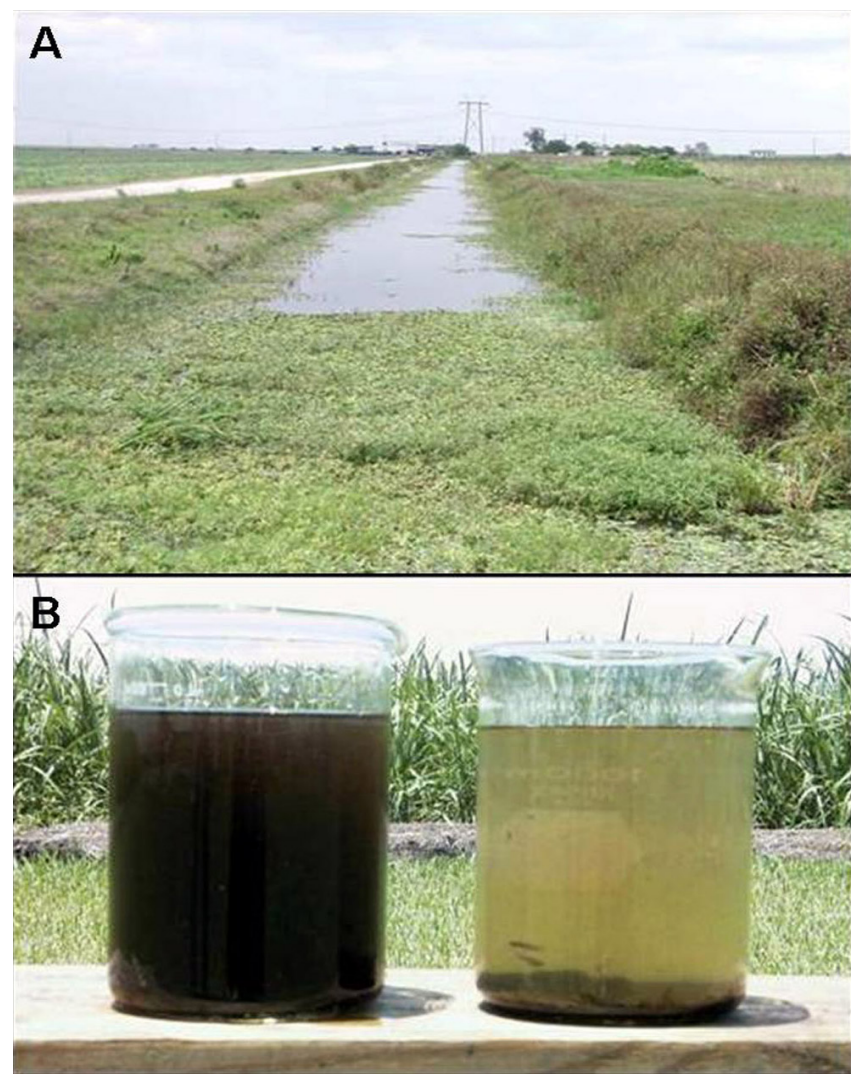

Figure 1. Algunos de los efectos de altos niveles de fósforo en las aguas de drenaje A) proliferación de vegetación acuática y B) aumento en la cantidad de sólidos en suspensión y turbidez.
La reducción de los niveles de oxígeno puede tener un efecto dañino en los peces y otras formas de vida acuática, causando una reducción de la biodiversidad y limitando el valor recreacional de los recursos naturales. La implementación de estrategias de manejo que maximizan la retención de $\mathrm{P}$ en las fincas y que también eviten el derramamiento accidental o directo de fertilizantes fosfóricos en el agua, resulta en la reducción significativa de las cantidades de $\mathrm{P}$ expulsadas de las fincas a través de las aguas de drenaje.

Algunas recomendaciones útiles para reducir la occurencia de derramamientos de fertilizantes incluyen:

- Estacionar los contenedores con fertilizantes, camiones y los aplicadores de fertilizantes lejos de las zanjas de campo y los bancos de los canales de las fincas.

- Estacionar los contenedores con fertilizantes y los camiones en terrenos nivelados (evitar pendientes que conduzcan hacia el agua en los canales; Figura 2).

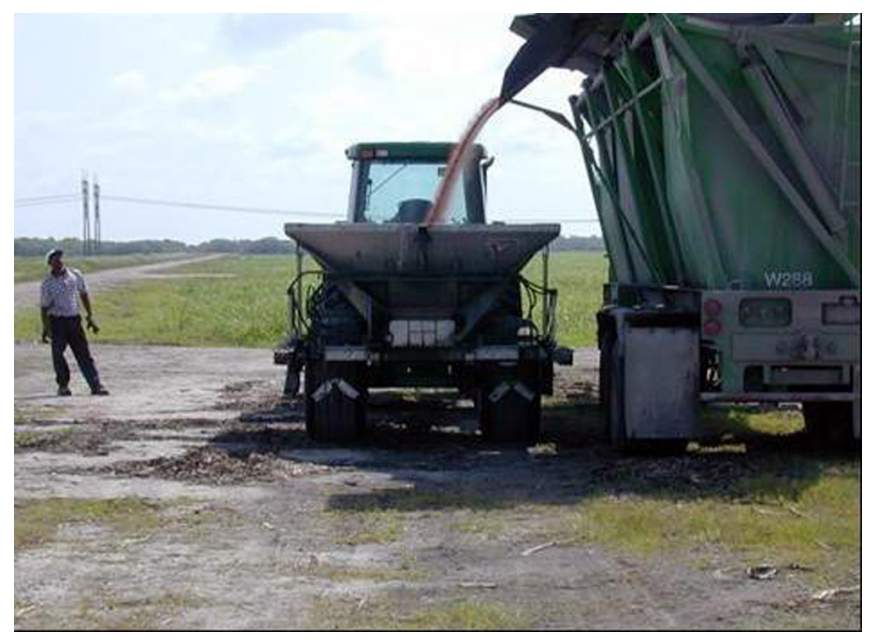

Figure 2. Sitio de cargamento de fertilizantes en terreno nivelado y lejos de zanjas abiertas y bancos de canales.

- Limitar el número de lugares usados para transferencia/carga de fertilizantes dentro de cualquier finca con el fin de facilitar los esfuerzos de inspeccionar / revisar estos lugares para encontrar derramamientos de fertilizantes no reportados (Figura 3). 


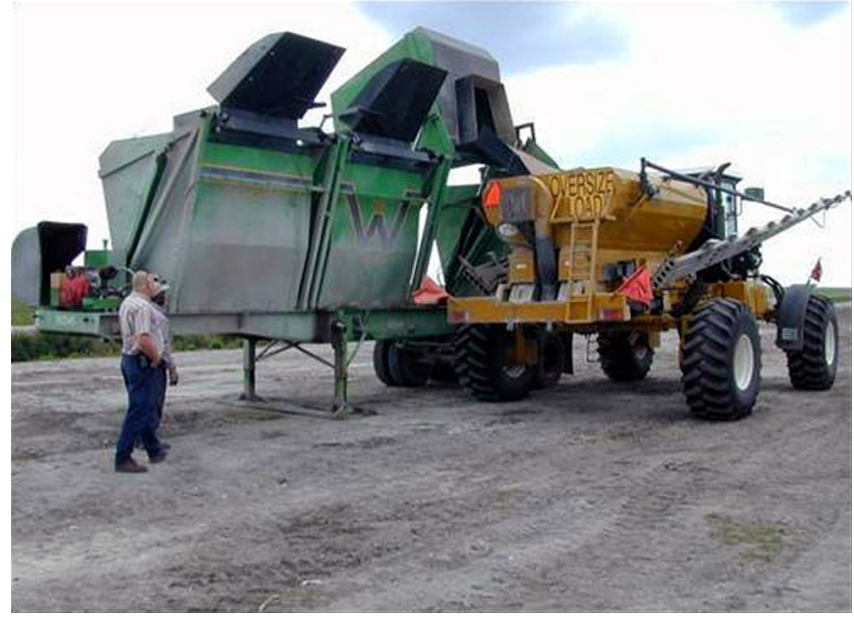

Figure 3. Inspección de un sitio de cargamento de fertilizantes.

- Los tanques con soluciones de fertilizantes deben ser lavados en el campo, y el agua usada para lavar debe ser aplicada directamente a los cultivos. Los tanques nunca deben ser lavados directamente, o vaciados directamente en corrientes de aguas, canales, otros cuerpos de aguas, $\mathrm{o}$ en carreteras.

- Entrenar y registrar correctamente a todo el personal envuelto en el manejo de fertilizantes.

- Como regla, estacione el equipo de fertilizar SOLAMENTE en sitios donde están las rampas de cargar la caña de azúcar, puesto que estas áreas son grandes, planas y frecuentemente visitadas.

En caso que ocurra un derramamiento de fertilizantes, los siguientes pasos deben ser tomados:

- Contener los derramamiento en carpas colocadas debajo de los camiones y aplicadores que reciben el fertilizante.

- Tener baldes y palas disponibles para la limpieza inmediata.

- Barrer "pequeños derramamientos"de los contenedores y camiones sobre las carpas y distribuir este material en el campo que estaba previsto ser usado.

- Como regla, todo el personal envuelto en el manejo y distribución de fertilizantes debe tener una copia de los procedimientos de operación exigidos para responder adecuadamente a un derramamiento de fertilizantes.

- El Procedimiento de Operación Exigido en el derramaminto de fertilizantes debe identificar el personal responsable de la limpieza y la cadena de comando para documentar las accciones de remedio tomadas.

\section{Reconocimientos}

Los autores están agradecidos por la ayuda finaciera de El Distrito de Protección del Medio Ambiente - Area Agrícola de los Everglades y El Departamento de Protección del Medio Ambiente del Estado de la Florida, fondos que permitieron la preparación de este documento así como la implementación de los estudios relevantes a las Mejores Prácticas de Manejo descritas aquí.

\section{Referencias}

Anderson, D.L., and A. Ceric. 1992. Reduction of phosphorus concentrations in agricultural drainage of the EAA by precipitation, coagulation, and sedimentation. Technical Report submitted to the Florida Sugar Cane League. University of Florida/IFAS, Everglades Research and Education Center, Belle Glade, FL.

BION Technologies. 1994. Bion Technologies Nutrient Management Systems. Technical Report submitted to the Sugar Cane Growers Cooperative of Florida. BION Technologies, New York.

Brown and Caldwell Consultans. 1993. Analysis and Development of Chemical Treatment Processes. Technical Report submitted to the South Florida Water Management District, West Palm Beach, FL.

Sharpley, A.N. 1999. Global issues of phosphorus in terrestrial ecosystems. P. 15-46. In K.R. Reddy et al. (ed.), Phosphorus biogeochemistry in subtropical ecosystems. Lewis Publ., Boca Raton, FL. 